\title{
La citoyenneté : sa définition, ses lieux et conditions d'exercice
}

\section{Tayeb Bouguerra}

\section{(2) OpenEdition}

1 Journals

Édition électronique

URL : http://journals.openedition.org/trema/1712

DOI : 10.4000/trema.1712

ISSN : 2107-0997

\section{Éditeur}

Faculté d'Éducation de l'université de Montpellier

\section{Édition imprimée}

Date de publication : 1 octobre 1999

Pagination : 69-72

ISSN : 1167-315X

\section{Référence électronique}

Tayeb Bouguerra, «La citoyenneté : sa définition, ses lieux et conditions d'exercice », Tréma [En ligne], 15-16 | 1999, mis en ligne le 01 octobre 1999, consulté le 01 mai 2019. URL : http:// journals.openedition.org/trema/1712 ; DOI : 10.4000/trema.1712

Ce document a été généré automatiquement le 1 mai 2019.

Trema 


\title{
La citoyenneté : sa définition, ses lieux et conditions d'exercice
}

\author{
Tayeb Bouguerra
}

1 Avant de répondre à la question de la définition que l'on peut donner à la citoyenneté, je souhaiterais faire une remarque globale qui porte sur l'émergence soudaine de la citoyenneté au cœur du champ pédagogique. L'éducation à la citoyenneté n'est pas en soi un phénomène récent puisque depuis le 10 septembre 1791, la mission dévolue à l'école républicaine est d'instituer (à travers les cours d'instruction civique ou de morale) aux valeurs de la République : liberté, égalité, fraternité, laïcité, tolérance. Dès lors se pose la question du pourquoi maintenant la citoyenneté: tout se passe comme si l'école d'aujourd'hui confrontée à un certain nombre d'événements (violence, montée de l'incivisme et des incivilités...) cherchait dans l'éducation à la citoyenneté un moyen de juguler le péril.

2 Le lancement des «Initiatives citoyennes à l'école pour apprendre à vivre ensemble » traduit sur le champ pédagogique ce besoin institutionnel qu'est la citoyenneté. La mission assignée à l'école en matière de citoyenneté est clairement définie dans deux textes officiels : la note de service №97-216 du 10 octobre 1997 adressée au recteur de l'académie de Paris, aux inspecteurs d'académie, directeurs des services départementaux de l'éducation nationale aux chefs d'établissement, aux directeurs d'école qui inaugure le lancement des «initiatives citoyennes» et la circulaire $\mathrm{N}^{\circ} 98-064$ du 26 mars 1998. On rappellera que le thème de la quinzaine de l'école publique pour 1998 est «Ecole de la citoyenneté, école de la dignité ». C'est dire si l'éducation à la citoyenneté a à voir autant avec le politique que le pédagogique.

3 Quant à la définition de la citoyenneté, elle ne va pas sans poser un certain nombre de problèmes: ses acceptions sont nombreuses (citoyenneté juridique, statutaire, effective...), son aire sémantique et notionnelle fort complexe tant elle tient à la fois à la nationalité, à la nation, à la patrie, à la communauté de destin, au droit, voire aux représentations. Dans un ouvrage récent, Le dictionnaire du citoyen, le citoyen français est ainsi défini : «Un citoyen français est une personne physique de nationalité française titulaire des droits et soumise à des obligations». La citoyenneté est à la fois jouissance de la chose 
publique (la respublica) et participation active aux affaires publiques; la civitas est à la fois droit de cité et concitoyenneté. Dans son acception moderne la citoyenneté contemporaine a à voir avec les valeurs fondamentales que sont la démocratie, la liberté, la tolérance, la laïcité ; elle se veut active, vigilante, soucieuse du respect des droits de l'homme dans le monde, de la défense des opprimés et de l'expression de la solidarité. Quant à la citoyenneté scolaire entendue comme "apprentissage de la vie en collectivité", elle est essentiellement définie dans les textes officiels comme, apprentissage de la "prévention des incivilités et de la violence, apprentissage du respect du travail des élèves et des éducateurs pour combattre la violence et ceux des hommes et des institutions (les pompiers, la police, la justice) qui ceuvrent à la vie civile.» On notera ici la contiguïté citoyenneté, violence et institutions chargées du maintien de l'ordre.

La question "De quels facteurs dépend l'exercice de la citoyenneté ", donne à lire que la citoyenneté s'exerce effectivement ; dans les faits elle est plus ou moins active (puisqu'on parle de citoyenneté passive). La citoyenneté s'exerce d'abord dans le droit de vote qui fonde sa dimension juridique. Elle s'exerce dans des lieux publics privilégiés (mairies, hôpitaux, sécurité sociale, agence pour l'emploi). Son exercice implique des partenaires, maires, préfets, administrateurs, préposés des différentes administrations, agents de l'ordre... On notera au passage que c'est dans ce type d'espace que différents facteurs qui relèvent des images et représentations (traits ethnotypiques, noms propres à consonance étrangère, lieux de résidence...) peuvent parasiter l'exercice de la citoyenneté. Et quand à tous ces facteurs vient s'ajouter le déficit linguistique, on peut comprendre que, parfois, la citoyenneté soit mal vécue. L'exercice de la citoyenneté comporte une dimension essentiellement sociale et se caractérise par une action transitive sur autrui et sur la société (participer pour changer les choses: dénoncer, prendre position, manifester sa solidarité, etc). C'est pourquoi les savoirs scolaires à faire acquérir me semblent pouvoir être corrélés avec les comportements citoyens tels qu'ils peuvent être inférés des textes officiels portant citoyenneté. Il importe de rappeler que les textes officiels qui constituent ainsi la citoyenneté en nouveau savoir à enseigner proposent un véritable programme d'éducation à la citoyenneté : les compétences à développer en matière de citoyenneté sont nettement identifiées. Il est question de savoirs (organisés autour des principes fondamentaux constitutifs de la démocratie, connaissance des règles de morale, des droits et des devoirs des collégiens, réflexion sur les notions de tolérance, de laïcité et de racisme, de liberté, de justice), de valeurs (organisation de débat d'idées autour des notions de tolérance, laïcité, solidarité, racisme...), des pratiques (rédaction de charte, organisation de débats, visite aux malades, expression concrète de la solidarité avec les malades et les démunis).

5 La question de «l'éducation à la citoyenneté par la construction d'un rapport au langage » me paraît pouvoir être abordée par l'articulation citoyenneté / comportements citoyens. Appréhendé dans une perspective communicative, l'apprentissage de l'expression de la citoyenneté gagnerait à mettre l'accent sur la dimension sociale des échanges et notamment sur les composantes socioculturelle, pragmatique et stratégique: nature des interactions et choix des modèles discursifs à actualiser compte tenu de la spécificité des situations, du statut des interlocuteurs et des effets à produire. L'apprentissage de la tolérance, du respect de la parole d'autrui, du règlement d'un différend, de l'expression de la nuance, de l'atténuation de ses propos, passe d'abord par la maîtrise des règles socioculturelles qui régissent la prise de parole 
(modalités d'ouverture, de développement et de clôture des échanges, modes d'interruption, de passation des tours de parole...).

Dans son rapport au langage, la citoyenneté peut être abordée en termes d'acquisition des moyens lexicaux, morphosyntaxiques, sémantiques, notionnels que l'expression des comportements citoyens en situation scolaire et extra-scolaire peut exiger. Apprendre à dire son refus de la violence, à condamner un fait qui porte atteinte à la collectivité, à exprimer sa solidarité avec les démunis, son indignation ou sa colère passe par l'apprentissage des mots pour le dire (réalisations langagières des actes de parole, modalisation d'énoncés, évaluation et auto-évaluation, paraphrases discursives...).

7 Ce sont là autant de nouvelles compétences à développer, de nouveaux objectifs à assigner à l'enseignement apprentissage du français, de nouveaux contenus à prendre en charge (apprentissage de la négociation, de la résolution de problèmes, de l'expression modalisée du point de vue...). Aussi le traitement didactique de la citoyenneté passe-t-il, à l'instar de tout apprentissage, par un travail d'opérationalisation (analyse des besoins, formulation d'objectifs, détermination des contenus, tâches à résoudre...).

\section{Deux remarques en guise de conclusion}

D'un point de vue didactique, l'éducation à la citoyenneté, parce qu'elle fait entrer la vie en classe de langue, me paraît représenter une grande chance de renouvellement de la didactique du français langue maternelle. Son apprentissage renouvelle tout à la fois la conception de la langue et de la communication, le choix des supports didactiques (documents sociaux, banderoles, affiches, graffitis, écrits sociaux et non plus scolaires...), la nature des activités pédagogiques (dépassement des exercices formels, exercices de simple manipulation d'unités linguistiques qui focalisent sur le développement de la compétence linguistique et ignorent la compétence de communication), les modalités d'évaluation.

Il a été beaucoup question des modalités de définition de la citoyenneté, des institutions, des enseignants; mais nous avons très peu parlé du principal intéressé par l'apprentissage de la citoyenneté, à savoir l'élève. Cet élève qui est là, en classe, avec son identité, ses repères, son monde intérieur, ses représentations... L'apprentissage de la citoyenneté ne doit pas ignorer l'élève citoyen: éduquer à la citoyenneté, c'est aussi choisir de tout dire : les droits et les devoirs du citoyen, l'accès à l'exercice réel de la citoyenneté, les risques de sortie de la citoyenneté.

\section{RÉSUMÉS}

Cette contribution poursuit la réflexion sur la citoyenneté en tentant de faire un inventaire de ses conditions d'exercice, lieux d'exercice, types de partenaires, modes d'exercice, activités, moments seront tour à tour envisagés. 
This research paper further pursues this symposium's reflection on civic/citizenship issues by endeavouring to pass under review the conditions in which it is exercised, the places where it is exercised, the means by which it is exercised, the activities involved and the moments when it is exercised, each of which are considered one after the other.

INDEX

Mots-clés : citoyenneté, institution

Keywords : citizenship, institution

\section{AUTEUR}

\section{TAYEB BOUGUERRA}

Université de Montpellier III, équipe Dipralang 